\title{
Modeling Resource Sharing Dynamics of VoIP users over a WLAN Using a Game-Theoretic Approach
}

\author{
Edson H. Watanabe, Daniel S. Menasché, Edmundo de Souza e Silva, Rosa M. M. Leão \\ Systems Engineering and Computer Science Department \\ COPPE - Federal University of Rio de Janeiro, 21941-590 - Rio de Janeiro, RJ, Brazil \\ \{edson,sadoc,edmundo,rosam\}@land.ufrj.br
}

\begin{abstract}
We consider a scenario in which users share an access point and are mainly interested in VoIP applications. Each user is allowed to adapt to varying network conditions by choosing the transmission rate at which VoIP traffic is received. We denote this adaptation process by end-user congestion control, our object of study. The two questions that we ask are: (1) what are the performance consequences of letting the users to freely choose their rates? and (2) how to explain the adaptation process of the users? We set a controlled lab experiment having students as subject to answer the first question, and we extend an evolutionary game-theoretic model to address the second. Our partial answers are the following: (1) free users with local information can reach an equilibrium which is close to optimal from the system perspective. However, the equilibrium can be unfair; (2) the adaptation of the users can be explained using a game theoretic model. We propose a methodology to parameterize the later, which involves active network measurements, simulations and an artificial neural network to estimate the QoS perceived by the users in each of the states of the model.
\end{abstract}

\section{INTRODUCTION}

Resource sharing mechanisms and congestion control are fundamental problems in computer networks. Over the last two decades, a wealth of models [1] and mechanisms [2], [3] have been proposed for establishing how multimedia content can thrive in a network where users compete for bandwidth. At the same time, it is very unlikely that one of those mechanisms will be widely deployed (as is TCP). In fact, due to the complexity of the current network, both in terms of number of applications and competing developers and users, any solution would not be applicable in an interesting class of situations and the most likely scenario is that in which the adoption of any control mechanisms will continue to be entirely voluntary (a la UDP). For those reasons, in this paper we consider a network that does not implement any kind of resource sharing mechanism, the later being delegated to the applications and end users of the system and henceforth called end-user congestion control. The end-user congestion control is the only ubiquitous multimedia congestion control mechanism deployed in the current Internet.

More sophisticated incarnations of user-level congestion control appear in the context of voice over IP (VoIP). Certain tools such as Skype dynamically adjust the congestion control mechanism to cope with varying network conditions. Skype may chose, depending on firewall configurations, either UDP or TCP, and it also automatically selects the codec in order to provide the best possible quality to its users [4].
In its full generality, end-user congestion control is intrinsically related to the freedom of the users. Again in the context of VoIP, users can decide, for instance, between different tools (Googletalk, Skype, etc.). The QoS offered to the users (which crucially depends on the congestion control mechanism implemented by the applications) will determine the evolutionary success of one application over the other.

Given that end-user congestion control is present everywhere, we pose the following two questions:

1) what are the performance consequences of letting the users to freely choose their rates?

2) how to explain the adaptation process of the users?

In this work, we present the results of an experiment involving users (students) that access a wireless local area network (WLAN) in order to transmit voice over IP (VoIP) traffic. This may become a common scenario as wireless hot stops are spreading in cyber cafes and airports, and the number of users of tools such as Skype is fast increasing. (It was recently reported that the skype user population reached one hundred million users [5].) From here on, we refer to the experiment with students as the behavioral experiment.

The VoIP users considered in this paper share an access point (AP), and choose freely the rate at which they receive data from the network. The individual decisions of a user are taken with the single objective of maximizing the quality of voice she receives. We are interested in the convergence point of this system and in the dynamic process by which the users converge to this point.

One of our findings was that, quite surprisingly, in all the controlled laboratory experiments, using students as subjects, the aggregate rate adopted by the users as a function of time (obtained by summing the rates of the codecs selected by the users with time) was close to the effective capacity of the channel [obtained through measurements (Section V)]. The users with only local information were able to adapt dynamically to the network conditions and converged to efficient points from the global system perspective.

It is at least intriguing that this occurred, specially if the problem is analyzed under the context of the tragedy of the commons. The tragedy of the commons is a type of social trap that involves a conflict over resources between individuals (in our case, users) and the common good (the network). According to Hardin [6], the tragedy of the commons is a 
member of a set of "problems which don't have a technical solution".

In this paper we adopt that view, and we do not propose any technical solution to guarantee the future of the stability of the Internet. Instead, we rely on the preliminary experimental results to claim that stability is possible, even in the absence of network congestion control mechanisms, if the users (1) are allowed to freely enter and leave the network, and that they will do the later if the network congestion is too high; (2) can choose the rate at which data is transfered (3) do no tolerate low voice quality.

To support the above claims, we propose a novel methodology for better understanding the behavior of selfish users that compete for medium access in a WLAN. In particular, the methodology involves an evolutionary game-theoretic model [7], and here is extended to take into account (1) and (3). In summary, we provide the following partial answers to our initial questions:

- free users with local information can reach an equilibrium which is close to optimal from the system perspective. However, the equilibrium can be very unfair;

- the adaptation of the users can be explained using a game theoretic model. This naturally leads to a new question: how to parameterize the model? We use active measurements, NS simulations and an artificial neural network [8] to estimate the QoS perceived by the users in each of the states of the model.

The key contributions of this paper are the following: (a) we extend the model proposed by [7] in order to have enough flexibility to explain the behavioral experiments; (b) we propose a methodology to infer the parameters of the model; (c) we conduct a limited number of behavioral experiments and show how the model can be used to explain their results. Notice that we only performed a minimal amount of behavioral experiments, their main purpose being solely to indicate the tendencies of the users and to show that it is possible to use the extended model in order to explain the experiments.

\section{BACKGROUND AND RELATED WORK}

The main objective of Game Theory is to understand how players interact in a situation where there are conflicts of interests. One is interested in determining the choices that players will make when faced with a particular game. This is sometimes referred to as the solution of the game. There are many concepts of game solution, but here we'll adopt the well known Nash Equilibrium. The Nash Equilibrium is a self-enforcing equilibrium with the property that no player can improve his expected payoff by modifying unilaterally his strategy.

The idea of using game theory to study the congestion control problem is not new. Altman and Basar [9], for instance, consider the scenario where each user requests a desired quality of service before the game starts. They propose an optimal control mechanism to regulate the rates of the users according to the requested QoS. Here, however, we do not propose an optimal control mechanism, since we are interested in a scenario where there is lack of agreements. More recently, Honggang et al. [10] propose and study the TCP congestion control control game, where users can freely decide the number of TCP connections to use. They present both a positive and a negative result: they predict a network collapse if users cannot anticipate the impact of their actions, but the possibility of a satisfactory equilibrium otherwise. Here, however, we are interested in the transmission of multimedia, which generally involves UDP and is sensitive to delay and bursts of losses.

We consider a scenario analogous to that suggested by Key et al. [11] and studied by Menasché et al. [7] using evolutionary game theory. It consists of a completely asynchronous environment where greedy users select dynamically their transmission rates based solely on the perceived user level voice quality.

The mainstay of our work is behavioral game theory [12]. In general, game theory may be used as a normative theory to establish what the designers and users should do (such as in auction design) or as a descriptive theory that helps us understanding reality and inferring metrics such as fairness. The later is called behavioral game theory.

The standard approach of behavioral game theory (which we follow) consists of performing real experiments in order to identify the points to which players eventually converge. The researches establish that those are Nash equilibria and try to infer the motivations and incentives that lead users to converge to these points.

Two papers related to our work are those of Friedman and Huberman [13] and Friedman et al. [14]. They also created a controlled framework emulating Internet environments to perform experiments with human subjects. Our work differs from those as follows. First [13], [14] emulate the network conditions via software. We use a real wireless link to conduct our experiments. Second, in these works, a utility function (which is related to a monetary reward offered to the players) was established by the game designer. In our work the users dynamically adapt to the network conditions based exclusively on the instantaneous voice quality they experience. Finally, we extend a recently proposed model [7] and use it as the basis for our studies on the system dynamics.

\section{The GAME-Theoretic Model}

The model considered in this paper is an extension of that proposed by Menasché et al. [7]. Consider a scenario where $N$ users share a wireless access point (hot-spot) and are able to dynamically select the bit rate of the incoming voice traffic they receive. The interaction of the users is modeled by a symmetric strategic game defined as $\mathcal{G}=\langle N, \mathcal{S}, \mathbf{u}\rangle$, where $N$ is the number of users that share the access point and $\mathcal{S}$ is the discrete set of strategies available, that is, the set of joint codec plus FEC mechanisms that any application user can select from. Note that each combined codec-FEC produces a certain rate at which the application flow is received. $K=|\mathcal{S}|$, the cardinality of the set $\mathcal{S}$, is the total number of choices or, equivalently, transmission rates available. Let $\mathbf{s}=\left\langle s_{1}, \ldots, s_{N}\right\rangle, s_{i} \in \mathcal{S}$, be the vector of strategies chosen 


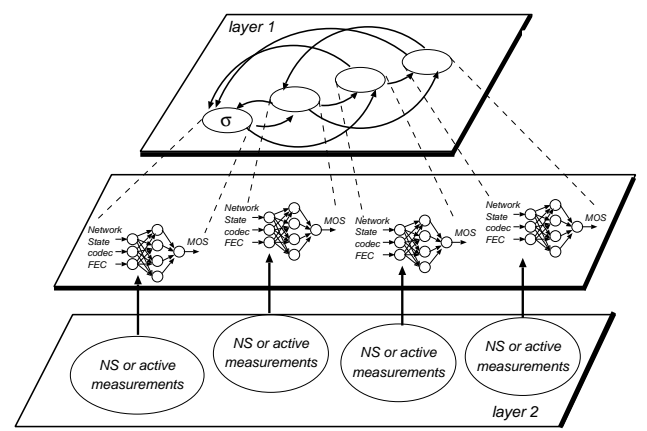

Fig. 1. The model.

by the players, also called the state of the population. The outcome of the game for a player adopting strategy $l$ is characterized by a real valued payoff (or utility) function $u_{i}(l, \mathbf{s})$. The utility function $u_{i}: \times_{j \in N} \mathcal{S} \rightarrow \Re$ determines the voice quality that user $i$ gets in each of the population states.

Let $\boldsymbol{\sigma}=\left\langle\sigma_{1}, \ldots, \sigma_{K}\right\rangle$ be the vector whose component $l$ indicates the number of players adopting strategy $l$ and $\mathcal{A}$ the set of all $(N+K-1) ! /[N !(K-1) !]$ such vectors for a given population of size $N$. Since we restrict ourselves to symmetric games [15, Section 9.2], the knowledge of $\boldsymbol{\sigma}$ is sufficient to characterize the utility function for each user. The utility function can also be expressed as $U_{i}(l, \boldsymbol{\sigma})$, where $U_{i}: \mathcal{A} \rightarrow \Re$. We chose to order and index the strategies increasingly with respect to the rate to which they are associated. We call $\sigma$ the state of the game.

As in [7], a continuous time Markov Chain (MC) $\mathcal{X}=$ $\{X(t): t \geq 0\}$ models the dynamic decision process representing how users choose their strategies. The state space of this $\mathrm{MC}$ is $\mathcal{A}$, defined above. The important distinctive characteristics between [7] and the model proposed here will be stressed next.

Associated with a state of the MC is a vector of utilities $\mathbf{U}(\boldsymbol{\sigma})$ where the $l$-th component is a measure of the voice quality for any user adopting strategy $l$ when $\sigma_{i}$ users are adopting strategy $i, i=1, \ldots, K$. One of the contributions of this work is the methodology to obtain $\mathbf{U}(\boldsymbol{\sigma})$ (Section IV).

Fig. 1 illustrates the model. On the upper layer, we model the behavior of the users and their strategy selection, while in the lower layer we model the wireless link. The first is accomplished through the MC $\mathcal{X}$, while the second can be obtained from measurements using a wireless access point and/or from simulation [we used both in this study (Section IV)]. Finally, there is an intermediary layer that maps the wireless characteristics (such as delay and loss rate) into the inferred QoS perceived by the users. The transition rates between any two states of the MC $\mathcal{X}$ are calculated from those inferred values of QoS, as we describe below.

The Dynamical Process of Strategy Adjustment:

Let $\boldsymbol{\sigma}^{(l, m)}=\left\langle\sigma_{1}, \ldots, \sigma_{l}-1, \ldots, \sigma_{m}+1, \ldots, \sigma_{K}\right\rangle$, be the state obtained from $\sigma$ after a user changes her strategy from $l$ to $m$. The transition rate from $\boldsymbol{\sigma}$ to $\boldsymbol{\sigma}^{(l, m)}$ is given by:

$$
\begin{cases}\sigma_{l} \Phi\left(U_{m}\left(\boldsymbol{\sigma}^{(l, m)}\right)-U_{l}(\boldsymbol{\sigma})\right) & \text { if } U_{m}\left(\boldsymbol{\sigma}^{(l, m)}\right)-U_{l}(\boldsymbol{\sigma})>0 \\ T & \text { and } U_{l}(\boldsymbol{\sigma})>\gamma \\ \epsilon & \text { if } U_{l}(\boldsymbol{\sigma})<\gamma \\ & \text { otherwise }\end{cases}
$$

We consider a scenario where users have imperfect information about the application level quality of service received (analog to the replicator dynamics [15, Chapter 9]). The larger the gain envisioned by a user when performing a certain change of strategy the larger the chances that the user will effectively modify the strategy in that way. Indeed, equation (1) indicates that the better a choice, the greater is the probability of changing in that particular way.

Users can make mistakes and transitions from $\boldsymbol{\sigma}$ to $\boldsymbol{\sigma}^{(l, m)}$ can occur even if the quality perceived at $\sigma$ is greater than that in $\boldsymbol{\sigma}^{(l, m)}$. This occurs with rate $\epsilon$, which is a parameter of the dynamic process. We assume that $\epsilon$ is smaller than the greatest difference of quality perceived by a user.

The above model premises are identical to those proposed by Menasché et al. [7]. In what follows we refine this model, explaining the extra parameters $T$ and $\gamma$. As shown in Section $\mathrm{V}$, those parameters give us enough flexibility to explain key aspects of the behavioral experiments performed.

We assume that users may not tolerate a very poor voice quality. A user that perceives a quality below a threshold $\gamma$ (the tolerance level) will explore different codec-FEC combinations at random in an attempt to obtain a better quality. Users experiencing a bad connection (i.e. $U_{l}(\boldsymbol{\sigma})<\gamma$ in equation (1)) try different alternatives at a rate $T$ (the exploration rate).

Eventually a user experiencing a bad quality may quit the system in order to retry later. We add one strategy to the model to encompass this decision. The rate at which such a user leaves the system is proportional to the difference between the current quality received and the tolerance level. Finally, we have to define the rate at which users reenter the system. For that purpose, we associate to each strategy $l>0$ a probability $p_{\text {retry }}^{l}$. The transition rate from state $\boldsymbol{\sigma}$ to state $\boldsymbol{\sigma}^{(0, m)}$ is given by $R \sigma_{0} p_{\text {retry }}^{m}$, where $R$ is the retrial rate.

For an example of the above, consider Skype users. Although they have no control of the receiving rate, Skype tries to automatically compensate for bad quality by varying the transmission rate [4], [16]. However, if the poor quality persists the only alternative may be to end the conversation and to retry later [17].

The model properties are the same as those described in [7]. In particular, when $t \rightarrow \infty$ and $\epsilon \rightarrow 0$ the states that are contained in singleton quasi-absorbing sets which receive non-negligible probability are Nash equilibria. However, not all the Nash equilibria have the same probability in steady state. Hence, if the system admits multiple Nash equilibria in some cases the model may be used to deal with the problem of Nash equilibrium selection.

Finally, a non-trivial issue is the characterization of the utility function $\mathbf{U}(\boldsymbol{\sigma})$. We must choose an appropriate model 
that accurately predicts the perceived quality for all users sharing the wireless access point for each combination of user strategies, that is for all possible values of $\sigma$. The work of [7] considered a bottleneck router and employed an $\mathrm{M} / \mathrm{M} / 1 / \mathrm{k}$ model to obtain performance metrics such as loss rate and delay (layer 2 of Figure 1) that would then be used as input to the E-model (intermediary layer of Figure 1). In this work we use the mean opinion score (MOS) as a voice quality measure and combine laboratory measurements plus simulations (layer 2 of Figure 1) as well as the random neural network (RNN) proposed in [8] and specially trained for our purposes (intermediary layer of Figure 1). A detailed discussion of the methodology to characterize the utility function is provided next.

\section{The Methodology to Parametrize the Model}

In this section we describe how the utility function of the analytical model proposed in Section III is obtained. The methodology is based on measuring the channel to obtain performance metrics (e.g., loss rate and jitter) and on using those to infer the user perceived voice quality. In order to generate the performance metrics we perform active measurements using the Tangram-II Traffic Generator [18] and simulations using the NS simulator [19]. To estimate the user perceived QoS we employed a Random Neural Network (RNN) proposed in [8]. Figure 2 summarizes the methodology. In what follows we describe, from right to left, the last two modules in the figure.

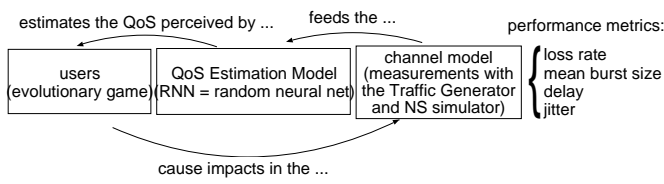

Fig. 2. The methodology adopted.

Inferring the Channel Model: We first need to estimate the wireless channel performance metrics (e.g., loss rate, delay and jitter) for all of the states of the MC model. For that we generated and transmitted voice flows over an wireless access point using the Tangram-II Traffic Generator.The Traffic Generator has two modules: a sender that injects data in the network according to the pattern established by the modeler and a receiver that computes statistics based on the information contained in the header of the packets.

We emulated with high fidelity the behavioral experiment (Figure 6). For each combination of user strategies, i.e., for each state of the proposed model, traffic (from the traffic generator) was injected into the wireless channel matching exactly the flows generated by VivaVoz. ${ }^{1}$ We measured the fraction of lost packet (PL), mean loss burst size (MLBS), jitter $(J)$ and one way delay (OWD).

Besides the measurements using the traffic generator, we also performed simulations with NS-2 (version 2.29). This

\footnotetext{
${ }^{1}$ VivaVoz, the VoIP tool that we used in the experiments with students, is open source and freely available as a part of the Freemeeting set of tools [20].
}

allowed us to corroborate the results obtained through emulation and also gave us flexibility to investigate a vaster number of scenarios. We considered the shadowing model for the channel and an infrastructured network with a DCF. Six nodes were disposed in a circle around the AP. We did not consider mobility. For each of the combinations of strategies considered we ran 30 turns of simulation of 100s each.

The QoS Estimation Model: As mentioned before we choose for the utility function of VoIP users the estimated Mean Opinion Score (MOS). The MOS is a subjective quality score that ranges from 1 (unacceptable) to 5 (excellent). Here we investigate how to obtain the MOS for each combination of strategies, once the performance metrics are measured.

The methodology of [8], called the Pseudo-Subjective Quality Assessment (PSQA), is well suited for our purposes. That work uses PSQA for quality assessment of one-way voice transmitted over the Internet and [21] employed the methodology focusing on interactive VoIP applications.

The PSQA methodology can be divided into three main steps [21]: (i) the choice of the quality-affecting parameters; (ii) the subjective assessment tests and; (iii) the selection, training and validation of a Random Neural Network (RNN). We use the RNN model defined in [21]. That model needs six parameters as input. Four of them are performance related measures: (a) loss rate, (b) mean loss burst size, (c) jitter and (d) one way delay; and the remaining two concern the encoding schemes used: (e) codec and (f) FEC.

Now we briefly describe the subjective tests performed by [21] to train the RNN model. The subjective tests involve a VoIP tool (the VoIP tool adopted was VivaVoz, the same tool used in the behavioral experiments) and a module to emulate the network conditions for each configuration (a modified version of the NetEm [22] Linux kernel was used). The tests were performed with subjects who had previously used VoIP tools. Two tasks were considered: taking turns counting up to 20 as quickly as possible, and a conversation based on a free topic, a picture or a scenario. At the end of the experiment, each individual gives a conversational quality score. Finally, the results are processed to obtain the MOS value for each configuration. A database with the MOS scores is then created in order to train and validate the RNN model.

Figure 3 illustrates two examples of the RNN output. In
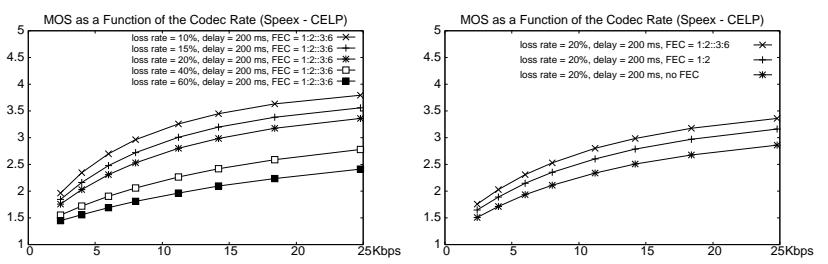

Fig. 3. The Random Neural Network output.

these figures FEC 1:2::3:6 are a combination of XOR FEC schemes of [23] and FEC 1:2 is the scheme of [24].

Figure 3(a) presents the MOS perceived by a user as a function of the codec rate for different values of the loss rate 
(the other four parameters were kept constant). In Figure 3(b) different FEC were adopted (the other four parameters were kept constant). As seen in the figure, for the parameter values used the higher the amount of FEC employed the greater is the MOS.

\section{RESUlTs}

In this section we report two sets of results. First, we present the outcome of the measurements that we used to parameterize the analytical model. Second, we show and discuss the results of the two behavioral experiments that we performed using students as subjects.

\section{A. Parameterizing the Analytical Model}

We start by measuring the effective capacity of the wireless channel, a IEEE802.11b/g . The access point was configured to support the nominal capacity of $1 \mathrm{Mbps}$ : the purpose of restricting the bandwidth is to increase the dispute for resources between users.

In order to compute this metric we injected data flows into the network, in order to emulate those generated by 6 VoIP users. We measured the goodput for each possible user rate and, in Figure 4, we plot the results in increasing order of the total aggregate rate. As expected, we can clearly distinguish two regions: one where the goodput increases approximately at a linear rate followed by saturation. When the aggregate rate injected into the system exceeds $180 \mathrm{kbps}$ the goodput oscillates around $160 \mathrm{kps}$ and we conclude that this is the channel effective capacity.

It is important to note that the effective channel capacity varies with the number of users. Since we perform experiments where users are allowed to terminate the VoIP connection, we also performed active measurements for obtaining the goodput with varying number of users. For up to 4 users, the aggregate flow generated do not saturate the channel, and the goodput increases according to the rates chosen. For 5 users the saturation occurs around $200 \mathrm{Kbps}$.

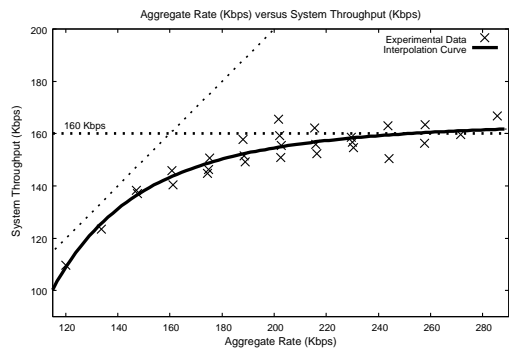

Fig. 4. The effective capacity of the system.

One fundamental issue is to understand the impact of the actions of a single user on the others. To this end, we did the following exercise: we set one of the hosts used in the measurements to generate traffic at a fixed rate $\lambda_{\star}$ while the others changed their rates progressively. $\lambda_{\star}$ was set to three levels: (i) low, 20 kbps (Speex 6 kpbs, FEC 1:2::3:6); (ii) medium, 33.6 kbps (Speex $14.2 \mathrm{kbps}$, FEC 1:2) and; (iii) high, 47.5 kbps (Speex 14.2 kps, FEC 1:2::3:6).

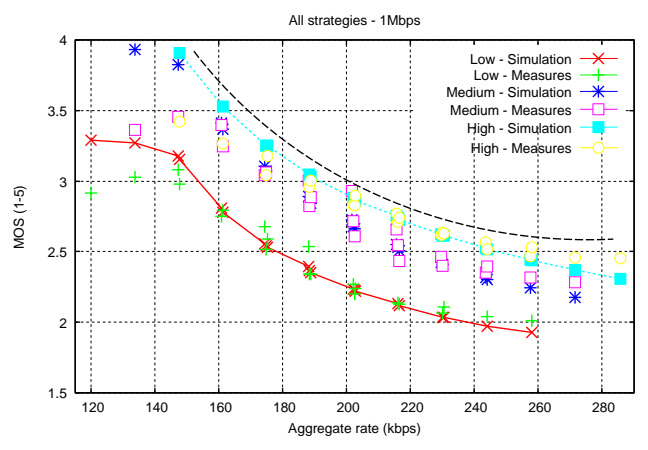

Fig. 5. MOS perceived by the users.

Figure 5 depicts the results of the performed measurements. Both the active measurements using the Traffic Generator as well as the NS-2 simulation results are plotted. In the $x$ axis we represent the system states in terms of the total aggregated rate at each (28 states, since we considered 3 rates and 6 hosts) while in the $y$ axis we represent the MOS using the random neural network inferred at the hosts with fixed rate $\lambda_{\star}$. Each state yields 6 metrics: 3 of them correspond to the simulation measurements obtained at a host that injects traffic using a low, medium and high rate while the 3 others represent the corresponding metrics obtained through active measurements. From the figure we observe that when the aggregate rate of the system increases the MOS perceived by a user adopting the fixed rate decreases. Note also that, in this scenario, the MOS perceived by a user adopting the medium rate is very similar to that adopting the high rate. Most importantly the figure shows the close agreement between the active measurements and the NS simulations, indicating that we can use the latter to parameterize the analytical model, instead of the costly active measurement approach.

\section{B. First Set of Behavioral Experiments: No bailing out}

1) Description: We selected six volunteers who were grouped into three pairs. The pairs were divided into two groups and each accommodated in one of the two rooms reserved for the experiments. Each player was assigned a machine with wireless support running a modified version of the VoIP tool VivaVoz [20] instrumented to record every action taken by its user. VivaVoz supports the Speex codec which provides 8 different rates that can be coupled with two levels of FEC, with overheads of 50\% and $100 \%$. (That is, the resulting transmission rate when FEC is used increases by $50 \%$ or $100 \%$, depending on the FEC scheme.) Figure 6(b) shows that a user receiving a VoIP flow can change dynamically the rate (the FEC and codec schemes) at which her peer is sending data. This can be easily performed from the tool's interface.

In one of the rooms we installed a commercial wireless access point (AP) IEEE802.11b/g (Us Robotics model USR5450, firmware 1.53b10). The network operated in the infra structured mode, with a DCF (Distributed Coordination Function) medium access protocol, without RTS/CTS frames and cryptography. The packets generated by VivaVoz are 


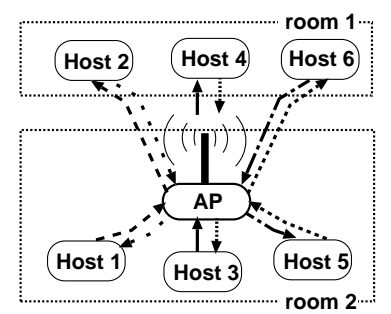

Fig. 6. The experiment topology.

TABLE I

StRATEgies AVAILABle tO EACH USER (RATES IN KBPS).

\begin{tabular}{|c|c|c|c|c|c|c|c|}
\hline Id & Codec & Redund & Rate & Id & Codec & Redund & Rate \\
\hline$\overline{11}$ & SPX 2.4 & None & 5.60 & $\overline{13}$ & SPX 11.2 & None & 14.40 \\
\hline 2 & SPX 2.4 & $1: 2$ & 11.60 & 14 & SPX 11.2 & $1: 2$ & 24.80 \\
\hline 3 & SPX 2.4 & $1: 2:: 3: 6$ & 16.00 & 15 & SPX 11.2 & $1: 2:: 3: 6$ & 33.50 \\
\hline 4 & SPX 4 & None & 7.20 & $\underline{16}$ & SPX 14.2 & None & $\underline{18.40}$ \\
\hline 5 & SPX 4 & $1: 2$ & 14.00 & 17 & SPX 14.2 & 1:2 & 30.80 \\
\hline 6 & SPX 4 & $1: 2:: 3: 6$ & 19.20 & $\underline{18}$ & SPX 14.2 & $\underline{1: 2:: 3: 6}$ & 41.50 \\
\hline $7^{*}$ & SPX 6 & None & 9.20 & $\overline{19}$ & $\overline{\text { SPX } 18.4}$ & $\overline{\text { None }}$ & $\overline{21.60}$ \\
\hline 8 & SPX 6 & $1: 2$ & 17.00 & 20 & SPX 18.4 & $1: 2$ & 35.60 \\
\hline 9 & SPX 6 & $1: 2:: 3: 6$ & 23.20 & 21 & SPX 18.4 & $1: 2:: 3: 6$ & 47.50 \\
\hline 10 & SPX 8 & None & 11.20 & $22 *$ & SPX 24.8 & None & 28.00 \\
\hline$\overline{11}$ & $\overline{\text { SPX } 8}$ & $1: 2$ & $\overline{20.00}$ & 23 & SPX 24.8 & $1: 2$ & 45.20 \\
\hline 12 & SPX 8 & $1: 2:: 3: 6$ & $\underline{27.50}$ & $24 *$ & SPX 24.8 & $1: 2:: 3: 6$ & 60.70 \\
\hline
\end{tabular}

sufficiently small so that fragmentation does not occur.

For the first set of experiments, once the connections have been established, users were requested to speak freely. During the conversation, each player was free to change the rate at which she received the voice traffic so as to maximize her perceived voice quality. The experiment ended when none of the participants changed their strategy during an interval of one minute or if the maximum time allocated for the experiment finished. We denote this experiment by interactive experiment since each pair of users freely talked (i.e., interacted). The interactive experiment was repeated 5 times (5 sessions).

A slightly different set of experiments, called non interactive experiment, was also repeated five times. In this set a prerecorded voice stream was sent from each participant to her peer. The other characteristics of the first set of experiments were kept.

Both sets of experiments were conceived to capture the following characteristics of a typical wireless network where users transmit VoIP traffic: (i) asynchrony, i.e., each user may change her codec and/or FEC mechanism at any given instant; (ii) incomplete information, i.e., each user knows only the rate that she uses to receive data. The users also know the number of strategies available to each. They do not know neither the rate that is being used to transmit data to the peer nor the rates that the other users are selecting; (iii) scarce resources, i.e., depending on the rate chosen by users, the decision may negatively impact the QoS of the others.

2) Experimental Results: Tables II and III show the rates selected by each user at the end of the non-interactive experiment and interactive experiment respectively. We call these the final rates. In these tables users $i$ and $i+1(i \in\{1,3,5\})$ belong to the same pair pair $(i+1) / 2$.

TABLE II

RESULTS FOR THE NON INTERACTIVE EXPER. (RATES IN KBPS)

\begin{tabular}{c|c|c|c|c|c|c|c}
\hline $\begin{array}{c}\text { Exp } \\
\#\end{array}$ & $\begin{array}{c}\text { Agregate } \\
\text { Rate }\end{array}$ & $\begin{array}{c}\text { User1 } \\
\text { rate }\end{array}$ & $\begin{array}{c}\text { User2 } \\
\text { rate }\end{array}$ & $\begin{array}{c}\text { User3 } \\
\text { rate }\end{array}$ & $\begin{array}{c}\text { User4 } \\
\text { rate }\end{array}$ & $\begin{array}{c}\text { User5 } \\
\text { rate }\end{array}$ & $\begin{array}{c}\text { User6 } \\
\text { rate }\end{array}$ \\
\hline 1 & 156.7 & 37.5 & 7.2 & 33.5 & 23.2 & 27.5 & 27.8 \\
2 & 152.5 & 41.5 & 16.0 & 17.0 & 33.5 & 27.5 & 17.0 \\
3 & 151.2 & 37.5 & 18.4 & 20.0 & 27.5 & 20.0 & 27.8 \\
4 & 160.5 & 37.5 & 27.5 & 20.0 & 23.2 & 23.2 & 24.8 \\
5 & 155.3 & 27.5 & 27.5 & 24.8 & 23.2 & 27.5 & 24.8 \\
\hline
\end{tabular}

TABLE III

RESULTS FOR THE INTERACTIVE EXPER. (RATES IN KBPS)

\begin{tabular}{c|c|c|c|c|c|c|c}
\hline $\begin{array}{c}\text { Exp } \\
\#\end{array}$ & $\begin{array}{c}\text { Agregate } \\
\text { Rate }\end{array}$ & $\begin{array}{c}\text { User1 } \\
\text { rate }\end{array}$ & $\begin{array}{c}\text { User2 } \\
\text { rate }\end{array}$ & $\begin{array}{c}\text { User3 } \\
\text { rate }\end{array}$ & $\begin{array}{c}\text { User4 } \\
\text { rate }\end{array}$ & $\begin{array}{c}\text { User5 } \\
\text { rate }\end{array}$ & $\begin{array}{c}\text { User6 } \\
\text { rate }\end{array}$ \\
\hline 1 & 135.2 & 37.5 & 7.2 & 20.0 & 33.5 & 17.0 & 20.0 \\
2 & 148.2 & 33.5 & 14.0 & 20.0 & 37.5 & 23.2 & 20.0 \\
3 & 148.6 & 37.5 & 5.6 & 20.0 & 37.5 & 23.2 & 24.8 \\
4 & 142.1 & 33.5 & 5.6 & 27.5 & 41.5 & 14.0 & 20.0 \\
5 & 160.0 & 41.5 & 9.2 & 20.0 & 47.5 & 17.0 & 24.8 \\
\hline
\end{tabular}

A striking observation from Tables II and III concerns the aggregated final rate adopted by the users. In all sessions this rate varies only from $135 \mathrm{kpbs}$ to $160 \mathrm{kbps}$. Since the effective network capacity was measured equal to $160 \mathrm{kbps}$ (Figure 4) this result indicates that the users, even without any coordination, converged to an efficient state where the network resources are almost fully utilized, but not overly saturated. The lack of a congestion control mechanism did not lead to a congestion collapse.

One may think it is not surprising that users converged to an efficient equilibrium where the network resources are fully utilized. However, note that users with only local information were able to adapt dynamically to the network conditions and converged to points where the aggregated rate (the sum the codec rates plus FEC overhead) selected by the users (Table I) is around the saturation knee of Figure 4 obtained from measurements. It is at least intriguing that this would occur.

The auto-regulation ability of the population may be credited to the fact that each user had to deal with tradeoffs while choosing strategies. Consider, for instance, the selection of the FEC scheme. Increasing the amount of redundancy permits the application to reconstruct a greater number of lost packets based on those that are effectively received. However, the choice involves a delicate tradeoff since the overhead associated with the redundancy may worsen the congestion problem (see for instance [25]). Now suppose that a user is receiving data through Speex $24.8 \mathrm{kbps}$ with 1:2 redundancy (Table I) and is having difficulty to understand her peer due to a high packet loss rate. This user may decide to decrease the rate of her codec to $18.4 \mathrm{kbps}$ without any redundancy and still obtain a better voice quality since this change helped to lower the overall congestion. In essence, these example illustrates some of the key issues related to the dynamic process of strategy selection.

Table II shows the results of the non interactive experiment. From this and Table I we can note that all users decided to 
protect their flows using a FEC scheme. Furthermore, if we compare each of the five experiments, the difference in rate between two distinct users decreased from experiment 1 to 5 . In the first experiment the rates differed by at most $30 \mathrm{kbps}$ while in the last they did not differ by more than $4 \mathrm{kbps}$. This resulted in a progressively fairer division of resources indicating an evolution of the strategic behavior of the users conducting the entire population to more uniform scenarios.

The results for the interactive experiment do not show the same trend as those for the non interactive experiment. In experiment 5, users 1 and 4 (Table III) used approximately $50 \%$ of the effective network capacity, while user number 2 consumed only $6 \%$ of the total capacity. This indicates that interactive users tend to be more aggressive than noninteractive users most likely because interactive applications demand for better quality. Indeed, some students involved in the experiments claimed that it was much more difficult to apprehend the meaning of an interactive conversation than to understand a pre-recorded tape. Our current rationale about it goes as follows. In the interactive experiment, assume that one user increases her received flow rate to try to improve the quality of the received voice. This act may decrease the voice quality perceived by her peer and result in the undesired effect of lowering the quality of the overall conversation. It indicates that the convergence process of interactive users is more sophisticated than that of the non interactive experiment.

3) Results from the Model: Here we analyze the strategic decisions of the users using the model presented in Section III. To this end, we selected three representative strategies, marked with bold face in Table I, among all the strategies available to the users during the behavioral experiment.

In Section V-A (Figure 5) we presented our results concerning the characterization of the utility function. In order to fully characterize the model parameters, however, we still have to infer $\gamma$ (equation (1)). That parameter has a direct impact on the Nash equilibria predicted by the model, which according to the behavioral game theory paradigm must match with the experimental results. In what follows, we use the results of the experiments presented in Section V-B2 to calibrate this last parameter of the model.

According to equation (1), one necessary condition for a state $\sigma_{e}$ to be a Nash Equilibrium is that the MOS perceived by all the users in $\boldsymbol{\sigma}_{e}$ must be greater than $\gamma$. As mentioned in Section V-B2, the aggregate throughput at the end of the behavioral experiments varied between $120 \mathrm{kbps}$ and $160 \mathrm{kbps}$ (Tables II and III). Figure 5 indicates that when the aggregate rate is in this range the MOS perceived by the users is greater than 2.7 . Therefore, we set $\gamma=2.6$ to satisfy the previously mentioned condition.

In Figure 7 we ordered the 28 configurations of the model increasingly with respect to the estimated mean MOS perceived by the users. We mark with circles the points of convergence observed during the behavioral experiment (Tables II and III) and with squares the Nash equilibria selected by the model. We observe two relevant facts. First, the users converged to points with high MOS. The loss of efficiency due to the absence of a central coordinator was not significant. Second, the calibrated model converged to a state where the mean MOS is high - more precisely, the point is in the same range as the convergence points experimentally observed. This shows that there is a reasonable agreement between the experiments and the model, despite its simplicity.

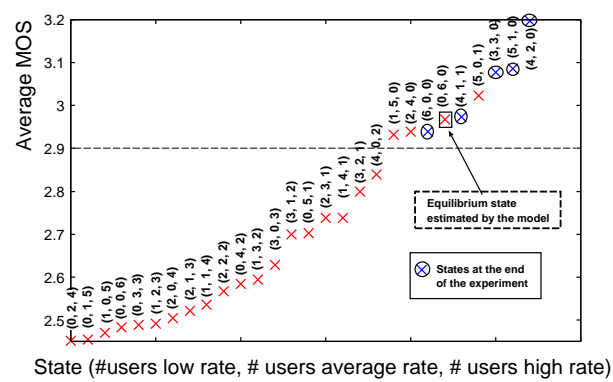

Fig. 7. Equilibrium States for the model and the experiment

Transient Analysis: Up to now we have only analyzed the game theoretic model in steady state. However, an important characteristic of the proposed model consists of the fact that it has different behavior depending on the time scale being considered. We refer to the dynamics that governs the first transition as the short term dynamics. Following Samuelson [26], we denote by medium term the period under which users are frequently changing their strategies; we refer to the long term as the time needed to reach a Nash equilibrium for the first time; finally, the steady state solution of the system is reached in the super long term.

For the sake of simplicity, to study the transient behavior of the system we start by setting the parameter $\gamma$ to zero. Our first goal is to identify the distinction between the long term and the super long term. In order to estimate the former, we could set $\epsilon=0$ and look for the mean time to absorption of the resulting MC. However, since the results obtained using this approach are very close to those presented in the left half of Figure 8 (which were generated using an ergodic MC with a very small $\epsilon$ ) we only show the later. The initial state in Figure 8 is set to $(0,5,1)$ (5 users using medium rate and one with high rate) and we plot 28 curves corresponding to the evolutionary dynamics of the probability mass of each of the states of the system. The three representative strategies selected for the transient analysis are marked with a star in Table I.

We can identify at least two regions in the left half of Figure 8: the medium term and the long term, the later starting approximately 100 time units after the system is initialized. Notice that, in the long run, there is a higher probability of finding the system in state $(0,6,0)$ (which confers a relatively high MOS to the users - Figure 7$)$ than in state $(0,0,6)$, which corresponds to the tragedy of the commons.

The right half of Figure 8 corresponds to the super long run. We see that although there is a high chance of having the system in state $(0,6,0)$ during the long run, in the super long run $(0,0,6)$ concentrates almost all the probability mass. 
It means that if the users do not establish a minimum threshold for the level of QoS that they can tolerate, the tragedy of the commons takes place. This is not the case, however, if we vary the tolerance level $\gamma$, as we consider next.

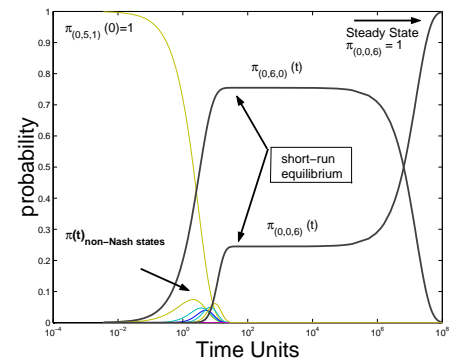

Fig. 8. Transient probabilities from the model, for $L=0, \epsilon=10^{-8}$.

In Figure 9 we divide the states into two sets: set $s_{1}$ is a singleton containing state $(0,6,0)$ while $s_{2}$ contains the 9 states listed in the legend. The state in $s_{1}$ confers a high MOS to the users while the states in $s_{2}$ correspond to lower levels of MOS. The curves represent the probability (y axis) of finding the system after 1000 units of time in the set $s_{1}$ or $s_{2}$, respectively, as a function of the initial state ( $\mathrm{x}$ axis). $\gamma$ is set to 2.5. As shown in the figure, there is a range of initial states that lead to a high probability mass for state $(0,6,0)$ at time 1000 . However, that range is still not very large. The scenario corresponding to $\gamma=2.6$ was briefly presented in Section V-B3 (although only in steady state). Finally, if we set $\gamma=3.0$ the situation drastically changes and with probability close to 1 the system is found in state $(0,6,0)$ after 1000 units of time, independently of the initial state.

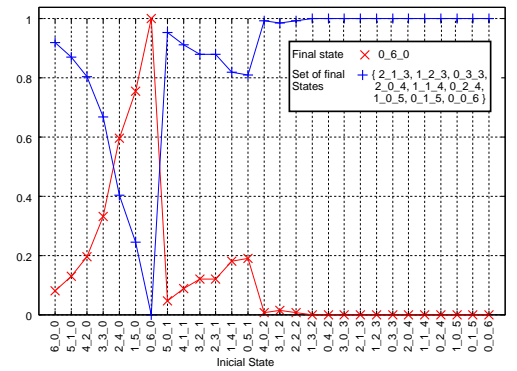

Fig. 9. State probabilities after 1000 units of time, for $L=2.5, \epsilon=10^{-8}$

\section{Second Set of Behavioral Experiments: Users allowed to bail out}

1) Description: For the next experiments, we selected 5 representative strategies from those in Table I (these are underlined in Table I) and limited the tool's user selection to those. However, we added one extra strategy to each user, namely the opportunity to quit the system and reenter later in case the user considered that the perceived quality of service was too low (Section III).
2) Experimental results: The evolutionary dynamics of the aggregated traffic is presented in Figure 10. One curve shows the aggregated traffic injected by the users in the channel as the function of the experiment time. We also kept track of the number of users active in the system (i.e., users adopting a strategy different from bailing out). The other curve shows the saturation throughput of the channel, that varies with the number of active users as indicated before.

Both curves of Figure 10 were obtained using the traces of the behavioral experiment. As mentioned in Section V-B1, those traces were collected using a modified version of the VivaVoz tool, instrumented to record every action taken by its user. From the traces of the strategies and Table I we infer the aggregate rate injected by the users into the channel. From the evolution of the number of active users (obtained through the traces) and the active measurements we derive the evolution of the saturation throughput of the system. We conclude that, in principle, the close matching of the curves that we see in Figure 10 could not have been anticipated. It must be an indication of the fact that the users can adapt to the varying network conditions. As we move from left to right in Figure 10 we see that the traffic curve tends to become a bit more far apart from the saturation curve. This indicates that the users tried to take full advantage of the available bandwidth, but at the same time were able to start an adaptation process to share the provided resources. Another interesting fact concerns the saturation throughput: it varied during almost all the experiment between $200 \mathrm{Kbps}$ and 150 Kbps which indicates that usually at least one user was bailing out so that those that continued actively using the network were able to experience an even higher QoS.

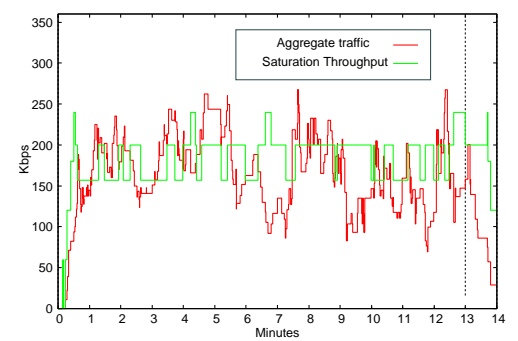

Fig. 10. Results of the second behavioral experiment.

3) Results from the Model: We now use the model presented in Section III to shed some light over the last behavioral experiment. We vary $\gamma$, from 2.6 to $4.0, R=1 / 30$ seconds (Section III) and $p_{\text {retry }}^{18}=p_{\text {retry }}^{24}=0.5$.

From the model we generated a number of sample paths to verify if the predicted behavior matched that from the behavioral experiments. We calculated the fraction of time the aggregates traffic was below the channel saturation point. For values of the tolerance $\gamma$ below 2.9, the model indicates congestion $35 \%$ of the time. However, as the user tolerance is set to values above 2.9 , the congestion sharply decreases reaching only approximately $10 \%$ and $5 \%$ for $\gamma=3.0$ and $\gamma=3.6$, respectively. As a conclusion, if users accept voice 


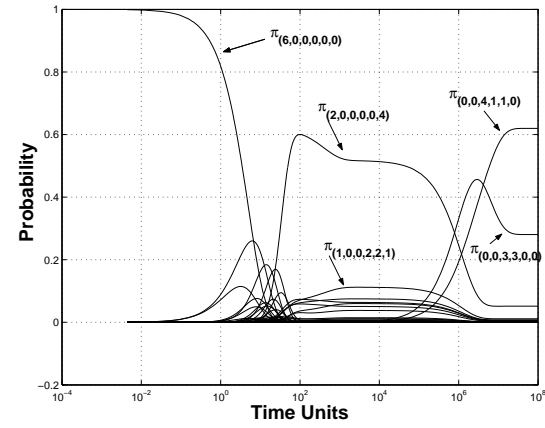

Fig. 11. Transient analysis from the model for the second experiment.

with very low quality, the system moves towards congestion and the tragedy of commons is likely to occur. But VoIP users hardly tolerate MOS levels below 2.8 and levels above 3.0 are acceptable. For higher tolerances values the users regulate their rates to levels below the channel saturation. It is remarkable that the model clearly shows this trend. In fact we have calculated the expected MOS values, averaged over all users, as a function of time from the behavioral experiment. Although this function is not shown due to space limitations a simple calculation shows that the expected MOS is $85 \%$ of the time above the 2.8 threshold (likewise, $15 \%$ of the time with poor quality meaning a congested network), corroborating the claim that users are able to adapt to reach acceptable voice quality levels avoiding the tragedy of commons scenario.

Figure 11 is analog to Figure 8 and shows the evolution of the probability mass as a function of time, given the initial state was that where all the users adopt the low rate. Again, we clearly identify the mean, the long and the super long terms. In the super long term the model was able to capture the fact that users with only local information adapted to the network conditions and converged to efficient equilibria.

\section{Summary AND CONCLUding REMARKS}

Given the wide dissemination of multimedia applications and overlay networks, as well as the trend to migrate many of the network functionalities to the application layer, the task of modeling congestion in computer networks tends to become increasingly challenging. In this paper we bring a new insight into that problem, borrowing ideas from behavioral game theory and presenting simulations, measurements and a theoretical models to explain the process through which free VoIP users adapt to the network conditions.

The behavioral experiments presented were performed with small populations. Although we were able to obtain consistent and relevant results using those experiments, we do not claim that it is straightforward to consider larger populations. Future work consists in devising scalable experiments in order to understand, for instance, the adaptation process of tens or even hundreds of attendees in a conference sharing a $56 \mathrm{Mbps}$ access point.

We study the end-user congestion control is the single ubiquitous mechanism of congestion control since it is intrinsically related to the freedom of the users. Users are free to enter and leave the system, as well as to choose the receiving voice traffic rates. Using a simple model we verify, for instance, that the tragedy of the commons is not a likely scenario provided that users have little tolerance to low voice quality. The experimental results corroborate this claim.

\section{REFERENCES}

[1] S. Floyd, J. Padhye, and J. Widmer, "Equation-based congestion control for unicast applications," in ACM SIGCOMM, Stockholm, 2000.

[2] Rejaie, Handley, and Estrin, "RAP: An End-to-End Rate Based Congestion Control Mechanism for Realtime Streams in the Internet," in IEEE INFOCOM, 1999.

[3] Nichols, Blake, and Baker, "Definition of the Differentiated Services Field in IPv4 Headers," 1998, IETF Request For Comments (RFC) 2474 and 2475.

[4] L. de Cicco, S. Mascolo, and V. Palmisano, "An Experimental Investigation of the Congestion Control Used by Skype VoIP," in WWIC, 2007.

[5] "Skype," http://share.skype.com/sites/en/2006/04/skype_hits_100_ million_users_c.html.

[6] G. Hardin, "The tragedy of the commons," Science Magazine, vol. 162, no. 13, pp. 1243-1248, 1968.

[7] D. Menasché, D. Figueiredo, and E. de Souza e Silva, "An evolutionary game-theoretic approach to congestion control," Performance Evaluation, vol. 62 (1-4), pp. 295-312, October 2005.

[8] S. Mohamed, G. Rubino, and M. Varela, "Performance evaluation of real-time speech through a packet network: a random neural networksbased approach," Perform. Eval., vol. 57, no. 2, pp. 141-161, 2004.

[9] E. Altman and Basar, "Multi-user rate based flow control," IEEE Transactions on Communications, 1998.

[10] H. Zhang, D. Towsley, and W. Gong, "TCP Connection Game: A Study on the Selfish Behavior of TCP Users," in IEEE ICNP, 2005.

[11] P. Key and D. McAuley, "Differential QoS and Pricing in Networks: Where flow-control meets game theory," in IEE Proceedings Software, 1999, pp. 177-182.

[12] C. Camerer, Behavioral Game Theory. Princeton, NJ: Princeton University Press, 2003.

[13] D. Friedman and B. Huberman, "Internet Congestion: A Laboratory Experiment," in ACM SIGCOMM Workshop of Practice/Theory of Incentives and Game Theory in Net Systems, 2004, pp. 177-182.

[14] E. Friedman, M. Shor, S. Shenker, and B. Sopher, "Asynchronous Learning with Limited Information: an experimental analysis," Games and Economic Behavior, vol. 47 (2), pp. 325-352, 2004.

[15] H. Gintis, Game Theory Evolving. Princeton, NJ: Princeton University Press, 2000

[16] Baset and Schulzrinne, "An Analysis of the Skype Peer-to-Peer Internet Telephony Protocol," in IEEE INFOCOM, Barcelona, Spain, 2006.

[17] Bu, Liu, and Towsley, "On the TCP-Friendliness of VoIP Traffic," in IEEE INFOCOM, Barcelona, Spain, 2006.

[18] E. de Souza e Silva, R. Leão, A. Rocha, F. Duarte, A. Silva, F. S. Filho, G. Jaime, and R. Muntz, "Modeling, Analysis, Measurement and Experimentation with the Tangram II Integrated Environment," in Int. Conf. on Performance Evaluation Methodologies and Tools, vol. 180. ACM Int. Conf. Proc. Series, 2006.

[19] "The network simulator ns-2." [Online]. Available: http://www.isi.edu/ nsnam/ns/

[20] Land, "VivaVoz," 2005, http://www.land.ufrj.br/tools/tools.html.

[21] A. P. C. da Silva, M. Varela, E. de Souza e Silva, R. M. M. Leão, and G. Rubino, "Quality assessment of interactive voice applications," UFRJ, Tech. Rep., 2006.

[22] Netem, "Netem home page," 2007. [Online]. Available: http: //linux-net.osdl.org/index.php/Netem

[23] D. R. Figueiredo and E. de Souza e Silva, "Efficient Mechanisms for Recovering Voice Packets in the Internet," in IEEE Globecom, 1999, pp. 1830-1837.

[24] J. C. Bolot, "End-to-end packet delay and loss behavior in the Internet," in Proceedings of the ACM SIGCOMM, September 1993, pp. 289-298.

[25] E. Altman, C. Barakat, and V. M. R. R., "Queueing analysis of simple FEC schemes for IP Telephony," in IEEE INFOCOM, 2001.

[26] L. Samuelson, Evolutionary Games and Equilibrium Selection. Cambridge, MA: The MIT Press, 1997. 